\title{
Enfermedad inflamatoria intestinal inespecífica
}

\section{Non-specific inflammatory bowel disease}

\author{
Christian Alberto Vargas Machuca-Carranza' \\ y Luis Ángel Rodríguez-Chávez ${ }^{2}$
}

Vargas Machuca-Carranza C, Rodríguez-Chávez L. Enfermedad inflamatoria intestinal inespecífica. $\mathrm{R}$ e $\mathrm{v}$ So $\mathrm{c} P$ e ru Me $\mathrm{d} \ln$ terna. 2020;33(3): II0-1 I 2.

https://doi.org/10.36393/spmi.v33i3.548

\begin{abstract}
RESUMEN
La enfermedad inflamatoria intestinal inespecífica hace mención al grupo de enfermedades en que al momento del diagnóstico no pueden clasificarse como enfermedad de Crohn o colitis ulcerativa, representa aproximadamente hasta el $15 \%$ de las enfermedades inflamatorias intestinales. Se presenta el caso de una paciente que inicio con dolor abdominal crónico que progresó a perforación intestinal por un proceso inflamatorio ileal, que luego de diversos estudios no se logró identificar la causa y que sumado a las características de la anatomía patológica se concluyó que se trató de un caso de enfermedad inflamatoria sin clasificar.
\end{abstract}

Palabras claves: enfermedad inflamatoria intestinal inespecífica, enfermedad inflamatoria intestinal.

\section{ABSTRACT}

Non-specific inflammatory bowel disease refers to a group of diseases which at the time of diagnosis cannot be classified as Crohn's disease or ulcerative colitis, and it represents approximately up to $15 \%$ of inflammatory bowel diseases. We present the case of a patient who started with chronic abdominal pain who progressed to intestinal perforation due to an ileal inflammatory process, that it could not be identified a cause after many studies and, added to the characteristics of the pathological anatomy, it was concluded that it was a case of non-specific inflammatory bowel disease.

Key words: Non-specific inflammatory bowel disease, inflammatory bowel disease.

\section{INTRODUCCIÓN}

La enfermedad inflamatoria intestinal (EII) es un grupo de afecciones intestinales inflamatorias crónicas idiopáticas. Las dos categorías principales de enfermedad son la enfermedad de Crohn (CD) y la colitis ulcerosa (UC) que tienen características clínicas y patológicas distintas y

Médico residente de medicina interna. Hospital EsSalud de Alta Complejidad "Virgen de la Puerta", Trujillo, Perú.

2 Médico internista. Hospital EsSalud de Alta Complejidad "Virgen de la Puerta",Trujillo, Perú. superpuestas. ${ }^{1}$ Sin embargo, en varios casos, esta distinción no es posible en el momento del diagnóstico o seguimiento y estos pacientes están etiquetados como enfermedad inflamatoria intestinal sin clasificar, no quedando claro si ellos constituyen un subgrupo distintivo de EII o si se debe considerar que se encuentran en las primeras etapas de CD o UC. ${ }^{2}$

\section{PRESENTACIÓN DEL CASO}

Mujer de 50 años que ingresó a emergencia por presentar dolor abdominal difuso tipo cólico de un mes de evolución que fue aumentando de intensidad, y que el día de ingreso se asoció a vómitos de contenido alimenticio. Al examen físico lo resaltante fue el abdomen con distención moderada, ruidos intestinales disminuidos, doloroso a la palpación difusa, a predominio de hipogastrio, sin signos peritoneales.

En los exámenes, nada resaltante excepto gasometría con acidosis metabólica. La tomografía abdominal evidenció un proceso inflamatorio en íleo distal con neumoperitoneo (ver figura 1).

Ingresó a laparotomía exploratoria donde se evidencia neumoperitoneo, líquido intestinal libre, plastrón conformado por asa sigmoidea, ovario izquierdo y asa ileal a $25 \mathrm{~cm}$ de la válvula ileocecal, además de la presencia 


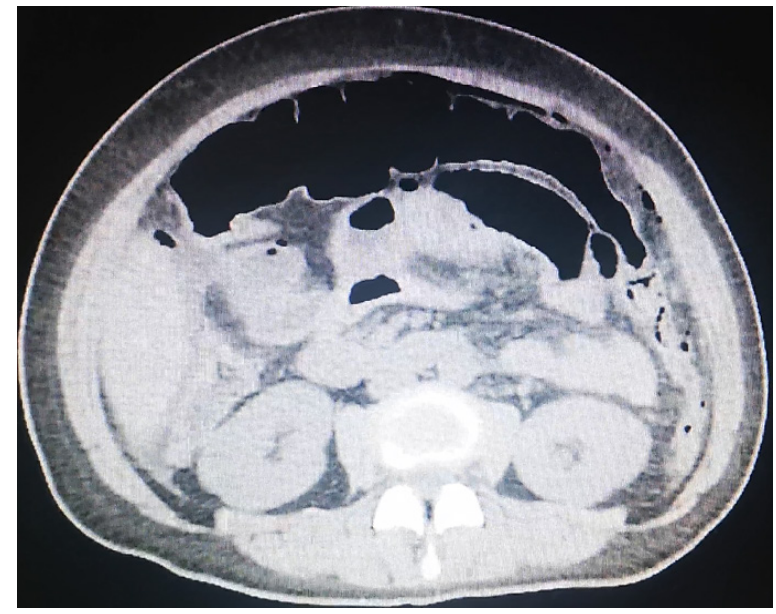

Figura I.Tomografía de ingreso, mostrando neumoperitoneo.

de una perforación a nivel de dicho plastrón con salida de material fecaloide.

En la anatomía patológica se observó: ileítis aguda inespecífica con necrosis ulcerativa de la mucosa asociada a foco de perforación y serositis aguda; la mucosa no necrótica del borde de sección con reacción inflamatoria crónica y sin evidencia de daño histológico crónico o granulomas; colon mostrando serositis aguda con compromiso focal de su pared por inflamación crónica activa.

La paciente presentó evolución favorable, portadora de ileostomía, fue dada de alta con dieta completa hiperproteica sin fibra, loperamida $16 \mathrm{mg} /$ día. Un mes después, presentó múltiples ingresos por emergencia por episodios de distensión abdominal, náuseas y disminución de la producción de ileostomía que se resolvía con tratamiento médico. Por persistencia de dichos cuadros fue hospitalizada para ampliar los estudios y posterior preparación para ileoscopía más toma de biopsia.

Endoscopía alta: esófago normal; gastritis erosiva; duodeno normal.

Baciloscopía (bK) en esputo, orina y heces negativas. VIH no reactivo. Hepatitis $\mathrm{B}$ y $\mathrm{C}$ no reactivos. Anticuerpos antinucleares y anti-DNA negativos. Perfil tiroideo normal.
$\mathrm{B}_{2}$-microglobulinas $1,7 \mathrm{mg} / \mathrm{L}$ ( $\left.\mathrm{VN}: 0,8-2,2 \mathrm{mg} / \mathrm{L}\right)$. Toxina de $C$. difficile negativo. Ca 125 7,5 U/ml (0-35 U/ml), Ca 19.9 7,26 U/ml (0-27 U/ml), Ca 15.3 4,3 U/ml (0-25 U/ml), CEA $0,76 \mathrm{ng} / \mathrm{ml}(0-6,5 \mathrm{ng} / \mathrm{ml})$.

Se intentó realizar la ileoscopía pero la luz intestinal fue pequeña tal que impedía la progresión del instrumento por abundante tejido de granulación, circundante y friable. La colonoscopia evidenció una mucosa de íleon distal con disminución de vellosidades y eritema difuso leve.

En la biopsia de íleon se observó mucosa ileal mostrando distrofia glandular asociada a reacción inflamatoria crónica inespecífica, levemente activa, sin granulomas ni abscesos crípticos, y no se encontró signos de neoplasia maligna (Figura 2). Se le realizó GeneXpert y cultivo para Mycobacterium tuberculosis que fueron negativos.

Las láminas de ambas biopsias fueron revisadas nuevamente por grupo de patólogos gastroenterólogos concluyendo finalmente que se trataría de una enfermedad inflamatoria intestinal sin clasificar por lo que, en el manejo médico, recibió mesalazina y corticoterapia con respuesta parcial al tratamiento, quedando en observación y seguimiento.

\section{DISCUSIÓN}

En el 2005, tras una revisión del tema, el Grupo de Trabajo de Montreal propuso que el término colitis indeterminada (IC) se reservara para los casos en los que se dispone de una muestra quirúrgica, mientras que el término enfermedad inflamatoria intestinal no clasificada (IBDU) debe usarse para pacientes que presentan características clínicas, endoscópicas e histológicas divergentes y para quienes no hay una muestra quirúrgica disponible. ${ }^{3}$

Hasta la fecha, hay muy pocos datos disponibles sobre la prevalencia de IBDU, algunos estudios de países europeos muestran que la frecuencia de IBDU es del $6 \%$ a $15 \%$ en adultos y $12 \%$ en niños con EII; además, para algunos expertos, la gran mayoría de los pacientes con IC/IBDU $(>80 \%)$ tendrán resultados funcionales a largo plazo idénticos a los de los pacientes con CU crónica, mientras que hay estudios que muestran que el $13 \%$ de los sujetos podrían permanecer sin clasificar después de un año de seguimiento. ${ }^{4,5}$

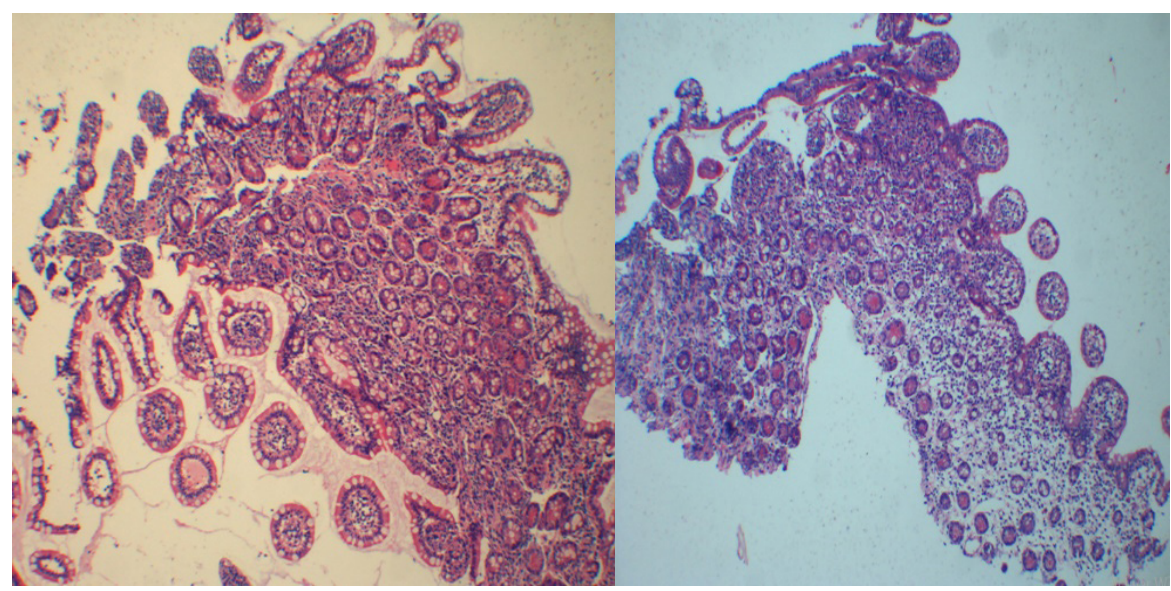

Figura 2. Mucosa ileal con reacción inflamatoria crónica inespecífica levemente activa. 
El diagnóstico correcto implica una estrecha colaboración entre el patólogo y el gastroenterólogo y se puede alcanzar con un seguimiento clínico cuidadoso que incluye una búsqueda de lesiones del intestino delgado y del tracto gastrointestinal superior con videocápsula enteroscópica, endoscopia del tracto gastrointestinal superior, otras técnicas de imagen y análisis de una nueva serie de biopsias. 6,7

Algunos marcadores serológicos podrían ser de utilidad en la distinción de la enfermedad inflamatoria intestinal; sin embargo, su uso sigue siendo controvertido. Dentro de estos se encuentran el anticuerpo anti-Saccharomyces cerevisiae (ASCA), positivo en el $80 \%$ de casos en CD, con sensibilidad y especificidad de $46,3 \%$ y $96,3 \%$; y, el anticuerpo citoplasmático anti-neutrófilo perinuclear atípico (pANCA) positivo en el $55 \%$ de casos en $\mathrm{CU}$, con sensibilidad y especificidad de $43,3 \%$ y 96,3 , respectivamente. En los casos de IBDU ambos anticuerpos son negativos. ${ }^{8,9}$

En cuanto a la terapéutica, la literatura rara vez se refiere al manejo de IBDU solo. Excepto por un suplemento alimenticio adecuado, hay una amplia gama de terapias disponibles que incluyen aminosalicilatos, corticosteroides, inmunomoduladores, productos biológicos y cirugía, aunque no está del todo claro qué tan bien IBDU responde clínicamente a ellos. ${ }^{10}$

\section{REFERENCIAS BIBLIOGRÁFICAS}

1. Bernstein CN, Eliakim A, Fedail S, et al. World Gastroenterology Organisation Global Guidelines Inflammatory Bowel Disease: Update August 2015. J Clin Gastroenterol. 2016;50(10):803-818.
2. Burisch J, Zammit SC, Ellul P, et al. Disease course of inflammatory bowel disease unclassified in a European population-based inception cohort: An Epi-IBD study. J Gastroenterol Hepatol. 2019;34(6):9961003. doi:10.1111/jgh.14563

3. Lopes S, Figueiredo P, Portela F, Freire P, Almeida N, Lérias C, et al Capsule endoscopy in inflammatory bowel disease type unclassified and indeterminate colitis serologically negative. Inflamm Bowel Dis. 1 de octubre de 2010;16(10):1663-8.

4. Prenzel F, Uhlig HH. Frequency of indeterminate colitis in children and adults with IBD - a metaanalysis. Journal of Crohn's \& Colitis. 2009 Dec;3(4):277-281. DOI: 10.1016/j.crohns.2009.07.001.

5. Zhou N, Chen W, Chen S, Xu C, Li Y. Inflammatory bowel disease unclassified. J Zhejiang Univ Sci B. 1 de abril de 2011;12(4):280-6.

6. Geboes K, Van Eyken P. Inflammatory bowel disease unclassified and indeterminate colitis: the role of the pathologist. J Clin Pathol. 2009;62(3):201-205. doi:10.1136/jcp.2008.059311

7. Joossens S, Reinisch W, Vermeire S, Sendid B, Poulain D, Peeters $\mathrm{M}$, et al. The value of serologic markers in indeterminate colitis: A prospective follow-up study. Gastroenterology. 1 de mayo de 2002;122(5):1242-7.

8. Fengming Y, Jianbing W. Biomarkers of Inflammatory Bowel Disease [Internet]. Vol. 2014, Disease Markers. Hindawi; 2014 [citado 23 de mayo de 2020]. p. e710915. Disponible en: https:// www.hindawi.com/journals/dm/2014/710915/

9. Ashorn S, Honkanen T, Kolho K-L, Ashorn M, Välineva T, Wei B, et al. Fecal calprotectin levels and serological responses to microbial antigens among children and adolescents with inflammatory bowel disease. Inflamm Bowel Dis. febrero de 2009;15(2):199-205.

10. Burisch J, Katsanos KH, Christodoulou DK, et al. Natural Disease Course of Ulcerative Colitis During the First Five Years of Followup in a European Population-based Inception Cohort-An Epi-IBD Study. J Crohns Colitis. 2019;13(2):198-208. doi:10.1093/ecco-jcc/ jjy 154

\section{CORRESPONDENCIA:}

Christian Alberto Vargas Machuca-Carranza.

christianvml6@hotmail.com

Fecha de recepción: 26-05-2020.

Fecha de aceptación: 20-07-2020. 